\title{
A telerehabilitation approach to delivery of constraint-induced movement therapy
}

\author{
Peter S. Lum, PhD; ${ }^{1-2 *}$ Gitendra Uswatte, PhD; ${ }^{3}$ Edward Taub, PhD; ${ }^{3-4}$ Phillip Hardin, MS; ${ }^{4}$ Victor W. Mark, MD $^{\mathbf{5}}$ \\ ${ }^{1}$ Hunter Holmes McGuire Department of Veterans Affairs Medical Center (VAMC), Richmond, VA; ${ }^{2}$ Biomedical \\ Engineering, Virginia Commonwealth University, Richmond, VA; ${ }^{3}$ Department of Psychology, University of Alabama \\ at Birmingham, Birmingham, $A L ;{ }^{4}$ Birmingham VAMC, Birmingham, $A L ;{ }^{5}$ Department of Physical Medicine and \\ Rehabilitation, University of Alabama at Birmingham, Birmingham, $A L$
}

\begin{abstract}
We have developed a device called AutoCITE (Automated Constraint-Induced Therapy Extension) that automates the intensive training component of constraint-induced (CI) movement therapy, also known as CI therapy. This study evaluated the effectiveness of AutoCITE training in a telerehabilitation setting when supervised remotely and with only intermittent interaction with a therapist. Seven participants with chronic stroke trained with AutoCITE for $3 \mathrm{~h} / \mathrm{d}$ for 10 consecutive weekdays. The therapist supervised the training from a different room in the clinic using remote control of the AutoCITE computer and teleconferencing equipment when needed. Treatment gains on the Motor Activity Log were quite large ( $p<0.001, d^{\prime}=3$ ), while gains on the Wolf Motor Function Test and the Jebsen-Taylor Hand Function Test were large $\left(p<0.05, d^{\prime}>0.9\right)$. Gains were comparable in size with those previously reported for participants who received equal intensities of directly supervised AutoCITE training or standard one-on-one CI therapy without the device.
\end{abstract}

Key words: arm, automated treatment, CI therapy, hemiparesis, motor recovery, rehabilitation, robotics, stroke, technology, telerehabilitation.

\section{INTRODUCTION}

Several clinical trials have shown that the application of constraint-induced (CI) movement therapy, also known as CI therapy, in patients with chronic stroke with mildly to moderately severe motor impairment produces a large increase in the amount of use (AOU) of the more affected upper limb that transfers to the life situation [1-9]. CI therapy consists of three main components: (1) concentrated task-based training (usually by shaping [3]) of the more affected upper limb for many hours a day for a period of consecutive weeks, (2) a package of transfer techniques designed to affect generalization of treatment gains from the laboratory/clinic to the life situation, and (3) restraint of the less affected limb for a target of 90 percent of waking hours. Clinical implementation of the technique is hindered by the large amount of one-on-one therapist supervision needed during the training component of the therapy and the trend of decreasing reimbursable therapist-patient contact time. Even if the treatment

Abbreviations: ADL $=$ activities of daily living, ANOVA = analysis of variance, $\mathrm{AOU}=$ amount of use, AutoCITE = Automated Constraint-Induced Therapy Extension, CI = constraintinduced, FA = Functional Ability [scale], MAL = Motor Activity Log, MCID = minimal clinically important difference, $\mathrm{PT}=$ performance time, $\mathrm{QOM}=$ quality of movement, $3-\mathrm{D}=$ threedimensional, VA = Department of Veterans Affairs, WMFT = Wolf Motor Function Test.

* Address all correspondence to Peter S. Lum, PhD; Biomedical Engineering, The Catholic University of America, Pangborn Hall, Room 131, 620 Michigan Avenue NE, Washington, DC 20064; 202-319-5657; fax: 202-319-4287.

Email: $\underline{\text { lum@cua.edu }}$

DOI: 10.1682/JRRD.2005.02.0042 
were readily available in the clinic, transportation issues in rural areas would limit access to the treatment. A telerehabilitation approach to delivery of CI therapy would greatly decrease treatment cost and increase access for many patients who could benefit from it.

We have developed a device called Automated CI Therapy Extension (AutoCITE) that automates the training portion of CI therapy, thereby reducing the amount of therapist effort needed to provide CI therapy and potentially overcoming the key obstacle to widespread use of CI therapy [10]. In previous work, we established that inclinic AutoCITE training, when supervised 100 percent of the time by a therapist, is as effective as the one-on-one training from a therapist that is done in standard CI therapy [10]. A subsequent study showed no loss of efficacy when AutoCITE training was supervised only 50 or 25 percent of the time [11]. In this study, we simulated the use of AutoCITE in a telerehabilitation setting and tested the efficacy of AutoCITE training when supervised remotely and intermittently.

The potential impact of telerehabilitation approaches to movement training has been noted [12], but a review of the literature finds few examples of formal patient testing. Feasibility of remote retraining of arm movement in stroke patients was demonstrated with the Java Therapy software [13]. A participant trained at home using the computer mouse and keyboard as input devices, interacting with a Web-based library of games and progress charts. Tasks included fast-as-possible finger tapping and targeted point-to-point reaching movements with the mouse. The programs automatically recorded participant performance and sent this information over the Web to a central computer. Movement parameters of the participant improved over several training sessions.

In another laboratory, more formal patient testing was performed on a virtual reality-based telerehabilitation system [14]. In this system, the therapist in the clinic specifies a task such as moving the hand through a doughnut. A three-dimensional (3-D) image of a doughnut appears on the participant's computer screen. A magnetic tracker records the participant's arm movement and projects over the doughnut image the trajectories taken during the task practice. Five participants trained at home for an hour each day for 4 weeks. Gains on a motor impairment scale were noted after training.

In-clinic testing of a virtual reality-based hand training system has been reported [15]. The input devices used were the CyberGlove (Immersion Corporation, San Jose, California) for measuring digit movement during range-of-motion tasks, and the Rutgers Master II-ND (commercially unavailable) glove for simulating interactions with virtual objects. Four participants trained in the clinic for 2 hours a day, 5 days a week for 3 weeks. Gains in movement parameters were noted, and two participants had gains in the Jebsen-Taylor Hand Function Test [15]. In another study from this group, the intensity of training was altered to daily training for 3.5 hours a day for 2 weeks [16]. All three participants had gains in movement parameters, and two patients had gains in the Jebsen-Taylor Hand Function Test. This group has reported on a telerehabilitation version of this system that incorporates games and exercises (i.e., pegboard) [17]; however, no clinical testing has been reported. Transfer of treatment gains to the life situation was not assessed in any of the mentioned studies.

While these studies are promising, this article reports on the first implementation and testing of a telerehabilitation approach based directly on CI therapy. CI therapy is one of the few treatments for chronic stroke that has gone through extensive clinical trials and been proven efficacious for stroke rehabilitation. If remote AutoCITE training is as efficacious as CI therapy when supervised in person by a therapist, then telerehabilitation via AutoCITE or similar devices would be appropriate and allow costeffective application of the treatment and increased access to CI therapy for many stroke survivors.

\section{METHODS}

\section{AutoCITE}

AutoCITE incorporates a computer and eight task devices arrayed in a cabinet on four work surfaces (Figure 1). The computer provides simple one-step instructions on a monitor that guides the participant through the entire treatment session. Completion of each instruction is verified by sensors built into the device before the next instruction is given. The participant controls two push buttons that are used when choosing a task; they thus allow the participant to control the flow of the session. The linear actuator that moves the cabinet has an integrated potentiometer that measures the position of the cabinet and allows the computer to move the workstation to preset heights assigned to each work surface. Once a task has been chosen, the appropriate work surface is automatically adjusted in height and manually pulled out and locked over the participant's lap. 


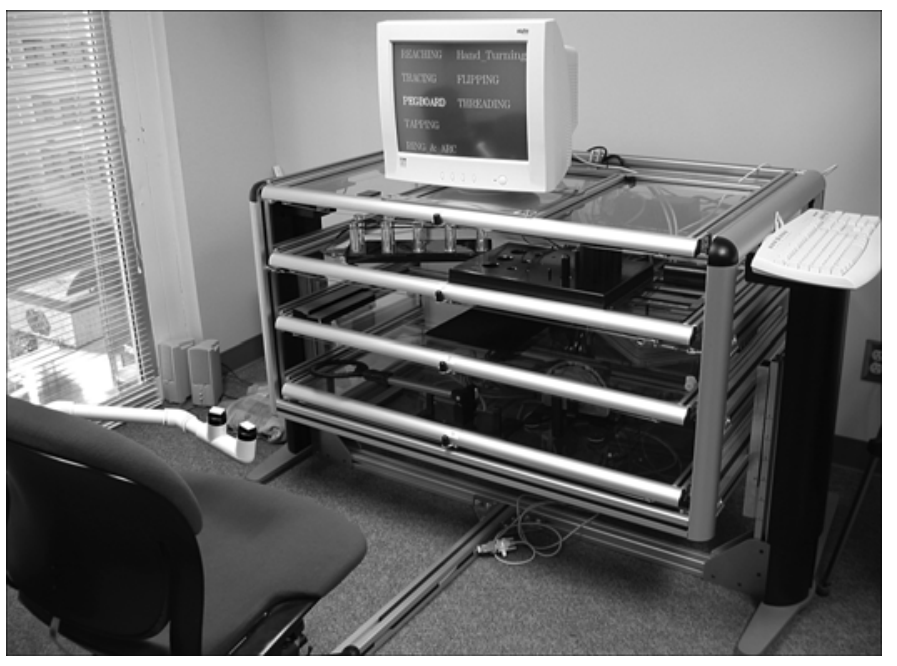

Figure 1.

AutoCITE with all four work surfaces pushed in cabinet. Entire cabinet automatically moves up or down until correct work surface is at lap level. Work surface is then pulled out and locked in place over subject's lap. Touch-screen monitor displays task selection menu. Two rectangular control buttons are on white rotating arm that is attached to left side of chair for right hemiparetic participant. Arm can be moved to right side of chair for participant with left hemiparesis.

The computer guides the participant through a set of ten 30-second trials. The participant is instructed to repeat a task as many times as possible within each trial. After each set of trials is completed, the task menu is displayed, which allows the participant to select the next task.

In this study, subjects performed all tasks before they started over again, but the subjects had control over the order in which the tasks were performed. Therefore, all subjects spent an approximately equal amount of time on each task.

Several types of results and performance feedback were provided. The time remaining on each trial is indicated on the computer monitor by a circle that is progressively filled in as time elapses, and an audible beep is produced when a repetition is completed. The number of successful repetitions is displayed after each trial in the form of a bar graph. Comments are provided by the computer based on current task performance: enthusiastic approval when performance improves and encouragement when it does not. Leaning forward with the torso during training is detected by a sensor that registers the flex of the chair and activates a buzzer when a criterion value is exceeded.

The AutoCITE tasks are based on tasks currently used in CI therapy and collectively address shoulder, elbow, wrist, hand, and finger function (Figure 2). In each case, sensors measure key aspects of the task and performance is automatically measured as the number of completed repetitions in $30 \mathrm{~s}$. The AutoCITE tasks are-

1. Reaching. This task involves successive tapping of a button just in front of the body and a target circle located on a touch screen. Task difficulty can be increased by moving the monitor farther away or higher.

2. Pegboard. The task is to move three pegs from a row of holes to a mirror-image row of same-sized holes on the other side of the board and then back again, etc. Peg sizes can be selected that require cylindrical grasp, three-jaw chuck, or thumb-index finger pinch.

3. Supination and pronation. Participants grasp a cylindrical handle that is mounted to a shaft-and-bearing assembly that allows forearm rotation about an axis. Participants rotate the handle back and forth between two specified angles. Difficulty is increased by requiring larger excursions of supination and pronation.

4. Threading. The task is to thread a shoelace through holes in a series of posts. This requires pushing the tip of the shoelace through a hole, reaching around to the other side of the post, regrasping the tip, pushing it through the next hole, and so on. The hole openings are funneled on one side of the posts but not on the other so that difficulty depends on the direction of threading.

5. Tracing. The touch screen presents large block letters that the participant has to trace with his or her fingertip or other portion of the hand. Difficulty is increased by decreasing the width of the letters, increasing the distance of the monitor from the participant, or increasing the height of the monitor.

6. Object flipping. The goal is to repeatedly flip over a rectangular block while keeping it on a work surface. The therapist increases difficulty by requiring the participant to use progressively smaller or larger blocks (depending on the nature of the participant's deficit).

7. Finger tapping. The task is to tap one finger as fast as possible while keeping the other fingers in contact with fingertip pads and the palm in contact with a palm rest. The therapist increases difficulty by moving the palm rest downward relative to the fingertip pads so that the task must be completed with the fingers in a more extended position. If the participant performs a tap and the palm is not in contact with the palm rest, the tap is not recorded and the audible beep associated 

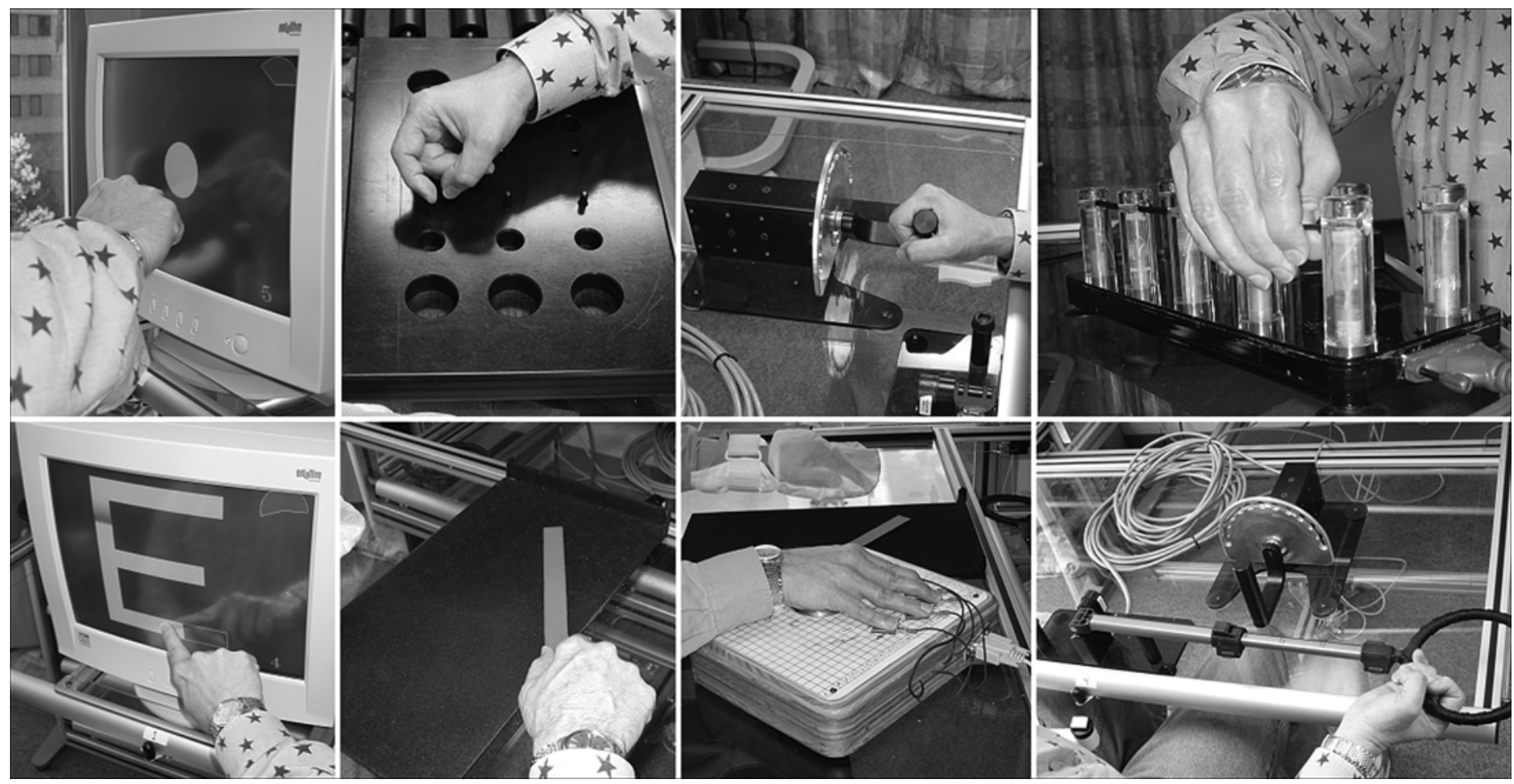

Figure 2.

AutoCITE task devices. Top row (left to right): reaching, pegboard, supination and pronation, and threading. Bottom row (left to right): tracing, object flipping, finger tapping, and arc-and-rings.

with a successful tap is not generated. The computer records the amount of time that nontarget fingers are off their respective pads and presents this information at the end of each $30 \mathrm{~s}$ trial.

8. Arc-and-rings. A ring is attached to the end of a long arm that is mounted to a shaft-and-bearing assembly that allows rotation of the arm in the frontal plane between two fixed mechanical stops. The task is to grasp the ring and rotate the arm of the device from one stop to another. Difficulty is graded by increasing the length of the arm of the device.

\section{Participants}

Seven patients with chronic stroke participated. All were greater than 12 months poststroke. All could extend at least $20^{\circ}$ at the wrist and $10^{\circ}$ at each of the metacarpophalangeal and interphalangeal joints and had greatly reduced use of the upper limb in activities of daily living (ADL). Participants were excluded if they had balance problems, excessive pain in any joint of the limb, uncontrolled medical problems, excessive spasticity, or cognitive problems as indicated by a score of less than 24 on the Mini-Mental State Examination [18]. The protocol was approved by the local institutional review board, and each patient signed an informed consent form.

Seven participants completed the training and posttreatment evaluations. Values throughout article are expressed in mean \pm standard deviation unless otherwise stated. The average age was $42.2 \pm 17.1$ years and the average chronicity was $9.9 \pm 17.7$ years. Three participants had right paresis and four had left paresis. Five participants were righthand dominant and two were left-hand dominant. Three males and four females were tested. The average baseline score on the Motor Activity Log (MAL) was $1.5 \pm 0.4$, while average scores on the Wolf Motor Function Test (WMFT) were $2.8 \pm 0.6$ for the functional ability (FA) scale and $3.9 \pm 2.4 \mathrm{~s}$ for the performance time (PT) scale. The average baseline score on the Jebsen-Taylor Hand Function Test was $43.7 \pm 28.4 \mathrm{~s}$.

\section{Procedures}

All participants were asked to wear a padded safety mitt on their less affected limb for a target of 90 percent of waking hours over a 2-week period [1]. A capacitive sensor recorded compliance with this procedure. This sensor was housed in the padded safety mit and activated 
a digital timer when the palm of the less affected hand was in contact with the mitt's surface. ${ }^{*}$ Participants also kept a diary of when they wore the padded safety mitt [19]. For 10 consecutive weekdays during this 2-week period, participants received training through shaping [3] using AutoCITE for 3 hours; testing and record-keeping activities usually took another half hour. To simulate a telerehabilitation setting, the therapist would set up the participant in AutoCITE and then retreat to a different room on the same floor. Videoconferencing equipment provided the therapist with video of the training activity and a two-way flow of audio between therapist and participant. This equipment was composed of two laptop computers (Apple Computer, Inc, Cupertino, California) that were connected via the hospital's local area Ethernet network - one laptop with the participant and the other in the therapist's location. Each laptop was equipped with a FireWire video camera (iSight, Apple Computer, Inc, Cupertino, California) with a built-in microphone. The dataflow was controlled with iChat AV software (Apple Computer, Inc, Cupertino, California). Once the session began, the therapist could control the amount of interaction by muting or activating the microphone on his end. A dedicated Ethernet line linked the AutoCITE computer to a second computer monitor and keyboard in the therapist's room. This allowed the therapist to see what was being displayed on the AutoCITE computer monitor and control the AutoCITE computer from his location.

The therapist was experienced in the delivery of CI therapy and used the following guidelines when remotely supervising the treatment:

1. At the end of one task and the beginning of the next task, the therapist would give the participant feedback on his or her performance including quality of movement (QOM) on the previous task and, if needed, instruct and coach the ensuing task. For example, the therapist did not attempt to limit internal/external rotation at the shoulder during the supination and pronation task. However, the therapist would verbally instruct the participant over the intercom if she or he were using obvious compensatory movement strategies.

\footnotetext{
* Taub E, Miltner W, Uswatte G, Splittgerber R, Jannett T. A device for objectively determining patient compliance with home training during Constraint-Induced Movement therapy. In: Uswatte G (Chair), Ambulatory monitoring of functional activity and treatment. Symposium conducted at the Annual Meeting of the American Congress of Rehabilitation Medicine. Tucson, AZ; 2003, October.
}

2. If a participant did particularly well on a trial, the therapist would reinforce the positive comments of AutoCITE (e.g., "Great work" or "First class"). If the participant struggled on a trial, the therapist would add encouraging words (e.g., “That’s fine. Just keep it up.”) or suggest strategies for improving performance.

3. If a technical problem occurred with the operation of AutoCITE, the therapist would communicate with the participant about the problem, either troubleshooting the problem with him or her or explaining what could be done or was being done to solve the problem.

4. If the participant used the audio intercom to address the therapist with a concern, question, or comment, the therapist would communicate with the participant. This happened very rarely; the therapist almost always initiated communication.

5. For the remainder of the treatment session, the therapist's microphone was muted so as to not distract the participant. The amount of time that the therapist's microphone was activated was recorded in the last five participants and was used to log the amount of therapistparticipant communication time. The intercom was kept on when the therapist left his room to interact with the participant in person.

Testing was carried out just before and after the intervention. The tests included the WMFT [20-21], the JebsenTaylor Hand Function Test [22], and the MAL [1]. The WMFT measures PT and FA on 15 tasks and the strength of shoulder flexion/elbow extension and grip in two tasks. The Jebsen-Taylor Hand Function Test measures PT for several hand tasks, such as picking up small objects and writing. The MAL is a structured interview that measures spontaneous use of the more affected upper limb in the life situation. It obtains information about 14 important ADL from such areas as feeding, dressing, and grooming, and provides scores on an AOU scale and a QOM scale. Correlations between the QOM and AOU scales at pre- and posttreatment were $>0.96$ as reported in Uswatte et al. [23]. The close association between the two scales is also discussed in a recent conceptual article [24]. Since the correlation between scores on these two scales is high, only the MAL QOM scale is reported here. The MAL was repeated 1 month after the end of treatment, and a long-term followup was conducted 6 to 12 months later. Details about the treatment and testing procedures can be found in the literature $[1,24-25]$. The original 14 -item version of the MAL was designed to assess higher-functioning subjects [1,26] and has recently been expanded to 28 items to better 
address lower-functioning subjects [27]. Since our study included only higher-functioning subjects, we used the 14-item version.

Score changes between test occasions were tested for significance with repeated-measures analysis of variance (ANOVA). Post hoc comparisons between sessions were done with paired $t$-tests, with a Bonferoni correction for multiple comparisons. The magnitude of the treatment effects was indexed using $d^{\prime}$, a within-subjects measure of effect size. By the standards of the meta-analysis literature, small, medium, and large $d^{\prime}$ values are 0.14 , 0.35 , and 0.57 , respectively [28]. Mean gains were compared with data from previous experiments on directly supervised AutoCITE training and standard CI therapy delivered by a therapist.

\section{RESULTS}

Participants showed significant gains in both arm function and real-world arm use after treatment. The repeated-measures ANOVA reported a significant effect for test occasion on the MAL $(p<0.001)$. Post hoc analysis found a significant pre- to posttreatment gain on the MAL $\left(2.1 \pm 0.7\right.$ points, $\left.p<0.001, d^{\prime}=3.0\right)$. The change in MAL scores between posttreatment and the 1 month follow-up was not significant (mean change $=0.0 \pm 0.2$, $p>0.9$ ). The MAL scores declined slightly between the 1 month and long-term follow-up, but this change was not statistically significant (mean change $=-0.3 \pm 0.9$, $p>0.9$ ). Scores on the WMFT also improved significantly at posttreatment. The mean change on the WMFT PT was $-0.9 \pm 0.9 \mathrm{~s}\left(p<0.05, d^{\prime}=1.0\right)$, and the improvement on the WMFT FA was $0.2 \pm 0.2\left(p<0.05, d^{\prime}=1.2\right)$. Jebsen-Taylor scores improved significantly after treatment (mean change $=-13.5 \pm 14.6 \mathrm{~s}, p<0.05, d^{\prime}=0.9$ ). By the standards of the meta-analysis literature, all the treatment effects can be considered large.

In each daily 3-hour training session, the therapist spent an average of 18.1 percent of the time communicating with the participant. Approximately once an hour, the participant encountered a problem that benefited from the therapist's presence; approximately 2 percent of the total training time was spent in direct face-to-face contact with the participant. Virtually all in-person contacts involved equipment problems (often dropping one of the test objects being manipulated); these will be corrected in an improved version of the present prototype device.
According to the compliance device housed in the padded safety mitt, participants wore the padded safety mitt for $88.5 \pm 7.7$ percent of hours in the laboratory and $64.3 \pm 19.3$ percent of waking hours outside of the laboratory. According to participant report, the padded safety mitt was worn $72.7 \pm 16.9$ percent of waking hours outside of the laboratory.

\section{DISCUSSION}

The gains in motor ability (WMFT) and real-world function (MAL) for the individuals treated using AutoCITE with remote supervision were comparable with the gains previously reported for chronic stroke subjects who received an equal amount of directly supervised AutoCITE training or standard one-on-one CI therapy [10-11] (Table). These previously tested individuals were recruited from the same pool and under the same inclusion and exclusion criteria and were treated and tested in the same laboratory as the participants in this study. A potentially confounding factor in this comparison is that the participants in our subject pool were significantly younger (age $=42.2 \pm 17.1$ ) than those in

Table.

Treatment gains on motor tests.

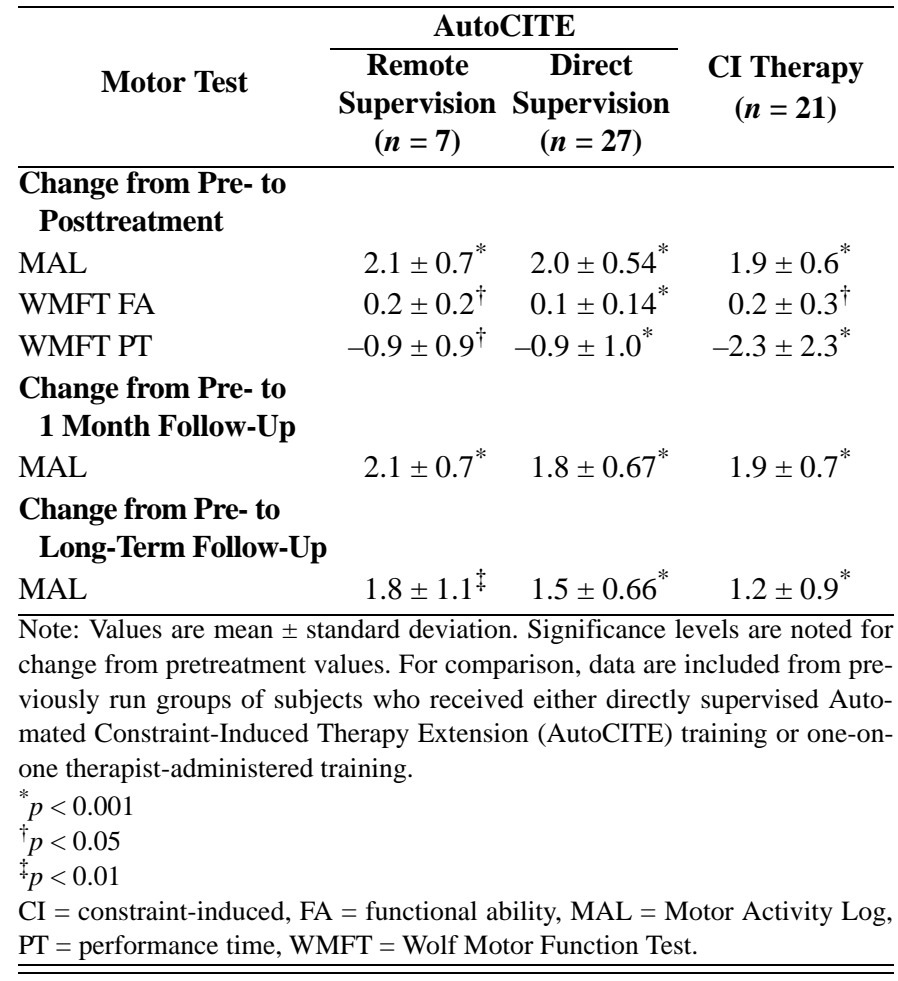


the group that received directly supervised AutoCITE training (age $=60.1 \pm 10.6$ ). The computerized knowledge of results might be more effective in younger populations who may be more familiar with computer technology compared with older participants who might be more motivated by one-on-one contact from therapists. However, when our participants were divided into younger and older groups, older participants ( $n=4$, age $=55.2 \pm 4.3$ ) scored the same as younger participants $(n=3$, age $=24.9 \pm 7.7)$ on the outcome measures $(p>0.4)$.

The MAL has been used extensively in CI therapy research and the minimal clinically important difference (MCID) has been defined by van der Lee et al. as values greater than 10 percent of full scale, or 0.5 points [29]. The MAL gains observed during remote training with AutoCITE were $2.1 \pm 0.7$ points, more than four times the MCID for this scale. On this basis, we conclude that the treatment gains were clinically significant.

Previous research has indicated that a key therapeutic factor of CI therapy is the concentrated training of the limb that patients are induced to complete. The rate at which training proceeded with the remote AutoCITE participants here was self-selected and greater than when a therapist controlled the rate based on patient preference and apparent fatigue. This finding indicates that AutoCITE has the capability to keep participants focused and motivated so that a high rate of practice can be maintained throughout treatment. Presumably, this high rate of practice is possible because AutoCITE provides immediate and detailed feedback on performance during and after each trial on an impersonal basis. Our clinical observation has been that in motivating participants' attempts to improve performance, this arrangement, contrary to our expectations, is more effective than AutoCITE training with a therapist present or when a therapist provides treatment without the use of such a device. Another important factor relates to the AutoCITE design, which promotes proper performance of the tasks and allows the task difficulty to be incremented in a manner similar to standard CI therapy. In these regards, CI therapy delivered remotely and through automation does not appear to limit the effectiveness of treatment.

Since these results are based on a simulated telerehabilitation setting, similar gains may not be achievable with a comparable remote device placed in the home. The AutoCITE room was a controlled environment without the distractions that might interrupt home training. The speed of the communication link between the AutoCITE and the therapist station was many times faster than is possible in home training, where the communication link will most likely be via regular telephone lines. Unless a broadband connection were available, the quality of the video feed at the therapist station will be poor compared with that which was available in this study. Nevertheless, we expect these factors to be minor as long as the targeted amount of training is achieved.

AutoCITE differs from other telerehabilitation devices in the following ways:

1. Perhaps most importantly, AutoCITE uses task devices, motivational feedback, and shaping rules that are based on CI therapy.

2. AutoCITE is similar to the Java Therapy approach. Although input devices for Java Therapy have not been developed other than the standard keyboard, mouse, and joystick, AutoCITE incorporates an array of task devices that mimic important movement components of ADL.

3. Virtual reality-based devices use sensors to record arm activity (i.e., CyberGlove, 3-D magnetic tracker), and the movement kinematics from these sensors are used to control a virtual image of the arm or hand. A video display presents the virtual arm or hand along with the task requirements. Actuated devices (i.e., Rutgers Master IIND glove) allow simulation of force interactions with virtual objects on the display. In contrast to this virtual reality-based approach, AutoCITE relies on an array of simple task devices. Instead of virtual objects, real objects are used. Instead of watching a virtual image of the arm, the subject watches his or her real arm. The key performance variables are measured via sensors built into the task devices. Thus, AutoCITE is much less costly compared with current virtual reality-based devices. Although the use of virtual reality-based systems may eventually prove to have advantages relative to devices such as AutoCITE, this remains to be demonstrated. Commercial implementation would favor the simplest and least-expensive device that facilitates the required training.

A potential limitation of the AutoCITE setup is that participants perform all tasks while sitting and above waist level. Although several common ADL are done within this spatial configuration, many ADL are done while standing or involve stooping. Thus, AutoCITE does not provide explicit training of upper-limb use in situations in which the whole body must be controlled, such as while standing. However, presumably when outside of AutoCITE and with the constraint mitt on the less affected 
limb, subjects will be forced to use the affected arm to perform activities that require use of the arm while standing and stooping. One should note that the MAL assesses transfer of function to the real-world environment in tasks carried out with the body in various postures.

\section{CONCLUSIONS}

The findings of this study justify continued investigation into telerehabilitation approaches for delivery of CI therapy. The current AutoCITE is designed for use in the clinic, and future work will focus on development of a portable version that can be used at home with remote supervision by therapists. Achieving the treatment outcomes of CI therapy in a home-based training protocol that incorporates remote supervision with only intermittent interaction with therapists would reduce the cost of the therapy and greatly expand access to CI therapy for stroke survivors.

\section{ACKNOWLEDGMENTS}

Dr. Lum is now with the Washington, DC, Department of Veterans Affairs (VA) Medical Center and with Biomedical Engineering at The Catholic University of America, Washington, DC.

This material was based on work supported by the VA Merit Review, grant B2480T.

The authors have declared that no competing interests exist.

\section{REFERENCES}

1. Taub E, Miller NE, Novack TA, Cook EW 3rd, Fleming WC, Nepomuceno CS, Connell JS, Crago JE. Technique to improve chronic motor deficit after stroke. Arch Phys Med Rehabil. 1993;74(4):347-54. [PMID: 8466415]

2. Taub E, Uswatte G, King DK, Morris D, Crago J, Chatterjee A. A placebo-controlled trial of constraint-induced movement therapy for upper extremity after stroke. Stroke. 2006;37(4):1045-49. [PMID: 16514097]

3. Taub E, Uswatte G, Pidikiti R. Constraint-Induced Movement Therapy: a new family of techniques with broad application to physical rehabilitation-A clinical review. J Rehabil Res Dev. 1999;36(3):237-51. [PMID: 10659807]
4. Miltner WH, Bauder H, Sommer M, Dettmers C, Taub E. Effects of constraint-induced movement therapy on patients with chronic motor deficits after stroke: A replication. Stroke. 1999;30(3):586-92. [PMID: 10066856]

5. Kunkel A, Kopp B, Muller G, Villringer K, Villringer A, Taub E, Flor H. Constraint-induced movement therapy for motor recovery in chronic stroke patients. Arch Phys Med Rehabil. 1999;80(6):624-28. [PMID: 10378486]

6. Sterr A, Elbert T, Berthold I, Kolbel S, Rockstroh B, Taub E. Longer versus shorter daily constraint-induced movement therapy of chronic hemiparesis: An exploratory study. Arch Phys Med Rehabil. 2002;83(10):1374-77. [PMID: 12370871]

7. Tarkka IM, Sivenius J, Pitkanen K. Improved motor performance after Constraint Induced Movement therapy in chronic stroke. Arch Phys Med Rehabil. 2001;82:1293.

8. Van der Lee JH, Wagenaar RC, Lankhorst GJ, Vogelaar TW, Deville WL, Bouter LM. Forced use of the upper extremity in chronic stroke patients: Results from a single-blind randomized clinical trial. Stroke. 1999;30(11):2369-75. [PMID: 10548673]

9. Platz T, Winter T, Muller N, Pinkowski C, Eickhof C, Mauritz $\mathrm{KH}$. Arm ability training for stroke and traumatic brain injury patients with mild arm paresis: A single-blind, randomized, controlled trial. Arch Phys Med Rehabil. 2001; 82(7):961-68. [PMID: 11441386]

10. Lum PS, Taub E, Schwandt D, Postman M, Hardin P, Uswatte G. Automated Constraint-Induced Therapy Extension (AutoCITE) for movement deficits after stroke. J Rehabil Res Dev. 2004;41(3A):249-58. [PMID: 15543442]

11. Taub E, Lum PS, Hardin P, Mark VW, Uswatte G. AutoCITE: automated delivery of CI therapy with reduced effort by therapists. Stroke. 2005;36(6):1301-4. [PMID: 15879335]

12. Winters JM. Telerehabilitation research: Emerging opportunities. Annu Rev Biomed Eng. 2002;4:287-320. [PMID: 12117760]

13. Reinkensmeyer DJ, Pang CT, Nessler JA, Painter CC. Web-based telerehabilitation for the upper extremity after stroke. IEEE Trans Neural Syst Rehabil Eng. 2002;10(2): 102-8. [PMID: 12236447]

14. Piron L, Tonin P, Trivello E, Battistin L, Dam M. Motor tele-rehabilitation in post-stroke patients. Med Inform Internet Med. 2004;29(2):119-25. [PMID: 15370992]

15. Boian R, Sharma A, Han C, Merians A, Burdea G, Adamovich S, Recce M, Tremaine M, Poizner H. Virtual realitybased post-stroke hand rehabilitation. Stud Health Technol Inform. 2002;85:64-70. [PMID: 15458061]

16. Merians AS, Jack D, Boian R, Tremaine M, Burdea GC, Adamovich SV, Recce $M$, Poizner $\mathrm{H}$. Virtual realityaugmented rehabilitation for patients following stroke. Phys Ther. 2002;82(9):898-915. [PMID: 12201804]

17. Popescu VG, Burdea GC, Bouzit M, Hentz VR. A virtualreality-based telerehabilitation system with force feedback. 
IEEE Trans Inf Technol Biomed. 2000;4(1):45-51. [PMID: 10761773$]$

18. Bleecker M, Bolla-Wilson K, Kawas C, Agnew J. Agespecific norms for the Mini-Mental State Exam. Neurology. 1988;38(10):1565-68. [PMID: 3419600]

19. Morris DM, Crago JE, DeLuca S, Pidikiti R, Taub E. Constraint-induced (CI) movement therapy for motor recovery after stroke. Neurorehabilitation. 1997;9:29-43.

20. Morris DM, Uswatte G, Crago JE, Cook EW 3rd, Taub E. The reliability of the Wolf Motor Function Test for assessing upper extremity function after stroke. Arch Phys Med Rehabil. 2001;82(6):750-55. [PMID: 11387578]

21. Wolf SL, Lecraw DE, Barton LA, Jann BB. Forced use of hemiplegic upper extremities to reverse the effect of learned nonuse among chronic stroke and head-injured patients. Exp Neurol. 1989;104(2):125-32. [PMID: 2707361]

22. Jebsen RH, Taylor N, Trieschmann RB, Trotter MJ, Howard LA. An objective and standardized test of hand function. Arch Phys Med Rehabil. 1969;50(6):311-19. [PMID: 5788487]

23. Uswatte G, Foo WL, Olmstead H, Lopez K, Holand A, Simms LB. Ambulatory monitoring of arm movement using accelerometry: An objective measure of upper-extremity rehabilitation in persons with chronic stroke. Arch Phys Med Rehabil. 2005;86(7):1498-1501. [PMID: 16003690]
24. Uswatte G, Taub E. Implications of the learned nonuse formulation for measuring rehabilitation outcomes: Lessons from Constraint-Induced Movement therapy. Rehabil Psychol. 2005;50:34-42.

25. Taub E, Crago JE, Burgio LD, Groomes T, Cook EW 3rd, DeLuca S, Miller NE. An operant approach to rehabilitation medicine: Overcoming learned nonuse by shaping. J Exp Anal Behav. 1994;61(2):281-93. [PMID: 8169577]

26. Uswatte G, Taub E, Morris D, Vignolo M, McCulloch K. Reliability and validity of the upper-extremity Motor Activity Log-14 for measuring real-world arm use. Stroke. 2005; 36(11):2493-96. [PMID: 16224078]

27. Uswatte G, Taub E, Morris D, Light K, Thompson P. The Motor Activity Log-28: Assessing daily use of the hemiparetic arm after stroke. Neurology. In press; 2006.

28. Cohen J. Statistical power analysis for the behavioral sciences. Hillside (NJ): Lawrence Erlbaum Associates; 1988. p. 19-74.

29. Van der Lee JH, Beckerman H, Knol DL, De Vet HC, Bouter LM. Clinimetric properties of the Motor Activity Log for the assessment of arm use in hemiparetic patients. Stroke. 2004;35(6):1410-14. [PMID: 15087552]

Submitted for publication February 10, 2005. Accepted in revised form June 13, 2005. 